\title{
Archéopages
}

Archéopages

Archéologie et société

$36 \mid 01 / 2013$

Exotismes

\section{Le goût des autres. Débat}

Propos recueillis par Catherine Chauveau

Jean-François Staszak, Jean Trinquier et Catherine Chauveau

\section{CpenEdition}

Journals

Édition électronique

URL : https://journals.openedition.org/archeopages/215

DOI : $10.4000 /$ archeopages. 215

ISSN : 2269-9872

Éditeur

INRAP - Institut national de recherches archéologiques préventives

Édition imprimée

Date de publication : 1 novembre 2013

Pagination : 48-55

ISSN : $1622-8545$

Référence électronique

Jean-François Staszak, Jean Trinquier et Catherine Chauveau, « Le goût des autres. Débat »,

Archéopages [En ligne], 36 | 01/2013, mis en ligne le 01 janvier 2015, consulté le 28 janvier 2022. URL : http://journals.openedition.org/archeopages/215; DOI : https://doi.org/10.4000/archeopages.215

(c) Inrap 
La notion d'exotisme a un ancrage historique précis. L'usage consacre l'adjectif exotique dans la langue française au XVI ${ }^{\mathrm{e}}$ siècle, puis le substantif exotisme au XIX ${ }^{\mathrm{e}}$, ces termes désignant ce qui vient d'ailleurs, mais avec une connotation positive. Peut-on transférer ce concept à d'autres époques? La question est d'abord de caractériser cet ailleurs. C'est un lieu lointain, mais par rapport à quoi ? Ensuite, comment, pourquoi, cet ailleurs est-il perçu comme bizarre? Et enfin, pourquoi aimer ce qui loin et bizarre?

\section{Jean-François Staszak}

est professeur au département de géographie et environnement de l'université de Genève. Ses recherches actuelles portent sur les représentations géographiques dans l'art et le tourisme, en rapport avec la question de l'altérité. Parmi ses dernières publications : «L'écran de l'exotisme. La place de Joséphine Baker dans le cinéma français », Annales de géographie, à paraître; « Planning prostitution in colonial Morocco: Bousbir, the quartier réservé of Casablanca », in P. Maginn and C. Steinmetz (eds), (Sub)Urban Sexscapes: Geographies and Regulation of the 'Sex Industry, London, Routledge, à paraître ; «The artist and the tourist: Gauguin in Tahiti», in Rakic T. and Lester J.O. (eds,), Travel, Tourism and Art, London, Ashgate, pp. 191-206, 2013 ; «La construcción del imaginario occidental del 'allá' y la fabricación de las 'exótica': El caso de los koi moko maoris », in Herniaux D. y Lidon A. (dir.), Geografia de los imaginerarios,Barcelone/Mexico, Anthropos/Universidad Autónoma Metropolitana Iztapalapa, pp. 179-210, 2012 ; «Limaginaire géographique du tourisme sexuel », L'Information géographique, 76, 2, pp. 16-39, 2012 ; «La fabrique cinématographique de l'altérité. Anna May Wong et les personnages de 'Chinoises' dans le cinéma occidental (1919-1940) », Annales de géographie, 682, 6, pp. 577-603, 2011.

\section{Jean Trinquier}

est maître de conférences à l'École normale supérieure et membre de l'équipe " Archéologie et philologie d'Orient et d'Occident » (UMR 8546). Ses recherches portent sur la représentation des lieux sauvages, notamment en tant que contremodèles, dans l'Antiquité. Parmi ses dernières publications : « La fabrique du serpent draco : quelques serpents mythiques chez les poètes latins », Pallas, 78, p. 221-255, 2008; « La mosaïque Barberini de Palestrina et limage de la faune éthiopienne dans l'Égypte lagide », in F.-H. Massa-Pairault et G. Sauron (éd.), Images et modernitéhellénistiques. Appropriation et représentation du monde d'Alexandre à César, Rome (Coll. ÉFR, 390), p. 23-60, 2007 ; « Hic sunt leones. La représentation des confins éthiopiens de l'Égypte dans la mosaïque Barberini de Palestrina », in F. Lecocq (éd.), L'Égypte à Rome, Actes du colloque de Caen 28-30 septembre 2002, Caen, Cahiers de la MRSH, 41, p. 339-382, 2005.
Propos recueillis par Catherine Chauveau. Merci à Christiane Descombin 

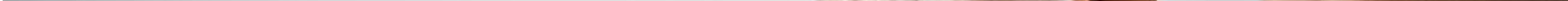
Jean-François Staszak Curieusement, dans un Occident qui prétend valoriser l'autre et l'ouverture à celui-ci, l'exotisme est plutôt déconsidéré aujourd'hui. Comme ce mot renvoie à l'artifice, à la superficialité et à l'inauthenticité, et qu'il n'est en rien un mot savant, la notion est méprisée ou ignorée par les spécialistes en sciences sociales d'une manière générale, alors qu'ils pourraient considérer le rapport de l'Occident au reste du monde comme un enjeu important. Et d'ailleurs, bien que la plupart de mes travaux concernent les composantes géographiques de l'altérité, je n'ai pas pensé pendant longtemps à les énoncer en terme d'exotisme. Puis le mot est devenu inévitable pour moi quand je me suis mis à travailler sur Gauguin, car il est clair que le moteur de sa démarche artistique, de ses voyages dans le Pacifique Sud, était l'autre et l'ailleurs. J'en suis venu à mettre ce concept au centre de mes recherches et à travailler sur ce désir des figures de l'autre. Mais est-il pertinent pour l'Antiquité de parler d'exotisme?

Jean Trinquier L'une des façons d'aborder le problème est l'enquête sémantique. Exotique est un mot d'origine grecque, l'adjectif exoticos, relativement peu employé en grec antique et ne désignant que ce qui est extérieur. En latin, perigrinus et exterus, nomment ce qui est étranger et ce qui vient de l'extérieur. Ces termes ne recouvrent nullement la notion moderne d'exotisme, c'est-à-dire cette appréciation positive de l'autre et de l'ailleurs. Il existe cependant un terme qui a le plus souvent une connotation négative, c'est l'adjectif barbaros en grec, barbarus en latin, pour nommer celui qui ne parle pas grec, mais aussi des produits ou des comportements étrangers, et même plus, rudes, sauvages. C'est donc plutôt le contexte qui permet d'appréhender le jugement de valeur sur l'autre et sur l'ailleurs, bien présent dans les textes antiques. JFS Lévi-Strauss pose l'ethnocentrisme comme un invariant anthropologique, toute société tendant à considérer ses valeurs et ses membres comme supérieurs à ceux des autres. L'attitude «normale» serait de mépriser, de déprécier l'autre et l'ailleurs. L'exotisme relèverait d'une attitude inverse, qui n'est pas nécessairement commune. Bien sûr, il n'est pas propre aux objets ou aux être exotisés, mais à un point de vue, un discours, un ensemble de valeurs et de représentations à propos de quelque chose, de quelque part ou de quelqu'un. Et de ce point de vue, les paysages, les visages, les valeurs etc. des autres ne sont pas juste des différences de normes mais des écarts, des carences, des outrances à «la» norme... En Europe, avant les grandes explorations, la représentation de l'ailleurs se fonde sur des récits, les textes classiques grecs, latins et les textes sacrés ${ }^{1}$. Cette connaissance ne repose donc pas sur l'expérience individuelle mais sur des images figées, répétées, d'autant plus frappantes qu'elles sont inquiétantes. Ce sont surtout des visions cauchemardesques d'un ailleurs dangereux peuplé de créatures à peine humaines mais elles laissent la place à quelques rêves heureux: les Îles fortunées, le Jardin des Hespérides, la fontaine de Jouvence, le Royaume du Prêtre Jean, et le Paradis! Tzvetan Todorov ${ }^{2}$ oppose ainsi la « règle d'Homère » à la « règle d'Hérodote »: L'Histoire du second démontre la supériorité des Grecs sur les Barbares tandis que l'Odyssée du premier révèle une fascination pour l'ailleurs, mystérieux et merveilleux à la fois.

JT Oui, l'ambivalence est manifeste dans le monde classique. Le territoire de l'altérité maximale est projeté sur les confins du monde connu, lequel comprend la Méditerranée et les terres qu'elle permet d'aborder. L'empire romain se conçoit comme un empire universel dont la force est sa capacité à maîtriser les confins, contrées sauvages contre-modèles du paysage cultivé et prospère garanti par Rome. Mais en fonction de l'objectif du discours, les textes présentent les confins soit comme des terres stériles, inutiles, que Rome ne se donne donc pas la peine de contrôler, soit comme des territoires domptés, subjugués, emplis de merveilles et de biens désirables ; des biens dont la valeur est en grande partie constituée par cette origine lointaine. Non seulement ils proviennent de contrées qui sont le lieu des raretés et des merveilles mais ils ont été acquis au prix d'immenses efforts pour surmonter de nombreux obstacles : la distance, les difficultés climatiques et géographiques, les animaux féroces et les populations incompréhensibles à tout point de vue et perçues comme hostiles. Cependant, dans certains textes dénonçant un présent civilisé marqué par la corruption et le déclin, cette altérité maximale est utilisée pour valoriser les peuples des confins, à qui l'on prête alors la simplicité des anciens Romains, ennemis du luxe et des vices. C'est un discours moraliste qui vilipende une intégration réussie, censée amollir et dégénérer, et magnifie une résistance obstinée à l'ordre romain. Et c'est un discours pour le moins paradoxal qui choisit pour modèle le contre-modèle! Cette valorisation n'est-elle possible que si ces peuples ne sont intégrés d'aucune façon ou bien, au contraire, parce que les récits et les images que l'on a d'eux les ont rendus plus proches et moins dangereux? JFS Je fais l'hypothèse que c'est dans un contexte impérialiste qu'on trouve l'autre attirant car si l'on n'est pas en mesure de le dominer et de le considérer avec une certaine condescendance, il est effrayant. L'exotisme est lié à la colonisation par l'Occident du reste du monde. Il semble très probable que l'impérialisme romain ait produit une situation semblable. Point d'exotisme en effet quand le monde est connu, parcouru, et que rien n'y apparaît plus étrange. Point d'exotisme non plus lors du premier contact qui rend l'autre si surprenant qu'il ne peut être que menaçant, repoussant. Pour qu'il soit charmant, l'autre doit apparaître non seulement loin de soi, 
Laltérité est, paradoxalement, l'un des ressorts de valorisation. 
géographiquement, historiquement ou socialement, mais également avoir déjà été abordé par le récit ou l'image, être déjà maîtrisé, assimilé. Se mêlent dans l'exotisme la place que se donne l'Europe puis l'Occident- en avance sur les autres continents, techniquement, économiquement, socialement, et le regard attendri qu'elle porte sur tel ou tel peuple figé dans un statut primitif ou premier. Disposer ainsi soi dans le présent dynamique et les autres dans un passé assoupi permet de les mettre à distance et de les absorber tout à la fois. On trouvera exotique ce dont l'étrangeté est acceptable - la danse des vahinés mais pas l'anthropophagie-, et ce que l'on peut considérer comme une expression antérieure ou inférieure de civilisation. En ce sens, l'exotisme est une forme de nostalgie, un voyage dans l'espace mais aussi dans le temps. L'anti-modernisme qui s'est développé en Europe à la fin du XIX ${ }^{\mathrm{e}}$ siècle, a nourri le primitivisme - le goût pour l'autrefois et l'exotisme - le goût pour l'ailleurs.

JT Cet attrait nostalgique pour une culture passée, c'est ce que les Romains ressentent pour le monde grec de l'époque classique et du début de l'époque hellénistique, et dans une moindre mesure pour leur Grèce contemporaine. C'est une altérité que les Romains se sont appropriée, qui est constitutive de l'identité qu'ils se sont construite à partir d'emprunts et de différenciations. Elle réserve à l'intérieur des normes de comportement romaines, une enclave de liberté, de douceur, de raffinement dans laquelle on peut échapper à la virilité rigoriste romaine. C'est une étrangeté intégrée, désirable à condition de rester cantonnée dans une sphère bien circonscrite, celle de l'otium privé.

JFS La question de l'exotisme aboutit fatalement à celle de l'érotisme car celui-ci relève du désir. Pour que l'étranger devienne exotique, il faut qu'il soit sorti de son contexte - dans lequel il est tout à fait normal - et replacé dans un autre où il devient bizarre et est transformé en objet de désir. Privé de son sens, défini par sa seule altérité, il est objet de contemplation et de consommation. Exporté et exhibé comme un substitut de l'ailleurs dont il provient ou observé sur place, par l'intermédiaire du savoir ethnographique, de la présentation du guide, du mitraillage photographique, il est réduit à un objet, livré à la curiosité et à la convoitise. Car s'il est lié à l'expansion occidentale moderne, l'exotisme l'est aussi à d'autres caractéristiques de cette société : la marchandisation, la science, l'encyclopédisme. Le marché de la prostitution a été alimenté par des femmes venues des colonies, dont le charme tenait pour partie à un imaginaire mêlant soumission et aventure. Dans les colonies, les femmes indigènes étaient perçues comme disponibles et excessivement sensuelle par rapport la vision qu'on avait de la femme européenne.

JT L'onomastique servile, et aussi celle des prostitués qui sont souvent des esclaves, fait bien apparaître cela. Les esclaves portent en général des noms ethniques - la Syrienne, par exemple - ou des noms qui font grec. La disponibilité sexuelle est souvent attribuée aux peuples du bassin oriental de la Méditerranée. La Grèce et l'Orient ne sont pas facilement distinguables dans les textes, puisque le Proche-Orient est hellénophone et en partie peuplé de populations grecques depuis longtemps. Ces contrées sont perçues comme le monde de la mollesse, du luxe, de la facilité, du plaisir, mais aussi de l'étranger assimilable. Les esclaves, hommes et femmes, valorisés comme beaux objets sexuels en viennent. Par exemple, l'un des biens de luxe appréciés faisant partie du décorum d'une table, est le beau serveur, le bel échanson; ce sont de jeunes éphèbes, grecs au sens large, dont le paroxysme de beauté est le puer alexandrinus, très jeune homme alexandrin aux traits caractéristiques : chevelure bouclée, grâce corporelle et puis une certaine forme d'esprit qui lui donne un peu de piquant.

JFS Produire des stéréotypes est le fait de l'exotisme ; stéréotypes des représentations et stéréotypes des productions. Parallèlement au commerce d'artefacts authentiques, rapidement limité, s'est développée une production de ces objets «typiques » évocateurs, au moins dans l'imaginaire de leurs propriétaires, de cet ailleurs désirable, et fabriqués en série n'importe où jusqu'à la nausée. Les modes des turqueries, chinoiseries, japonaiseries et autres objets aujoud'hui courants pudiquement nommés ethniques... se succèdent. Même devenu banal, l'exotique reste une valeur marchande, et chaque contrée de l'ailleurs est caractérisée par un ensemble d'objets, de mets, de paysages, d'ambiances etc. qui la définissent comme étrange, envoûtante, charmante... JT Rare, extraordinaire, faut-il ajouter pour l'Antiquité. La notion de confins pour le monde romain a cela de commode qu'elle offre à la fois un certain flou géographique et une ferme constance dans l'imaginaire qui lui est lié; un imaginaire développé sur le sentiment du merveilleux et du précieux. Et là on parle de produits bien réels comme la perle de l'océan indien ou de produits fantasmés, comme le sang-dragon. La première n'est pas, en soi, une merveille car le monde romain connaît les perles atlantiques mais il attribue une valeur supérieure à celles provenant de lieux quasi inaccessibles. Difficile de dire si elles étaient réellement de meilleure qualité. La description du sang-dragon se trouve dans divers types de textes tel le Périple de la mer Érythrée $e^{\mathbf{3}}$ très factuel, et d'autres, plus fabuleux, sur les produits lointains. Le sang-dragon est un colorant rouge, utilisé notamment par les peintres et nommé cinnabari. Ce cinnabari peut être soit le sulfure rouge de mercure, soit la résine d'un arbre endémique de l'île de Socotra, au large de la corne de l'Afrique dans l'océan Indien. L'autre nom de cette résine rouge était haima drakontion, c'est-à-dire le sang, en fait la sève, d'un arbre appelé drakontion, c'est- 
L'exotisme est aimable; il ne doit pas faire peur ou interroger.

Jean-François Staszak 
à-dire sève, de l'arbre dragonnier. Mais par une interprétation littérale du terme, cela est devenu « le sang du drakôn ». Un drakôn, dans l'Antiquité, ce n'est pas un dragon mais un serpent; dans le monde tempéré, une couleuvre, et dans le monde exotique, l'équivalent d'une couleuvre démesurément agrandie qu'est le python. Cette interprétation du nom repose sur l'une des nombreuses anecdotes animalières qui couraient sur la faune lointaine, à savoir l'antagonisme entre ces deux vedettes que sont l'éléphant et le python. On avait été tellement frappés par la puissance de ces pythons nouvellement découverts qu'on est allé jusqu'à penser qu'ils étaient capables de se mesurer avec succès à des éléphants. D'autre part, on avait été frappés par la présence de ces deux animaux en Éthiopie et en Inde. L'histoire est que le python s'enroule autour de l'éléphant pour aspirer son sang. L'éléphant exsangue, en s'écroulant, écrase et tue le python. De ces deux cadavres suinte le mélange des sangs. Non seulement la substance vient du plus loin des loins mais elle naît d'un duel entre deux animaux incroyables et dangereux, fascinants. Cela n'a pas de prix! Cette attraction pour les animaux exotiques est attestée déjà dans l'œuvre d'Hérodote, par exemple. L'extraordinaire élargissement des horizons créé par les conquêtes d'Alexandre a fait qu'on a pu voir et capturer ces créatures sans équivalents dans le pourtour méditerranéen. Les récits les concernant prennent fréquemment une tournure fabuleuse.

JFS Cette fascination pour les animaux exotiques a t-elle conduit, comme dans l'Europe moderne, à la constitution de zoos?

JT On a longtemps pensé, on a beaucoup écrit, qu'il y avait à Alexandrie un zoo, un peu perçu sur le modèle de la bibliothèque ${ }^{4}$. Ce qui est avéré, c'est un goût des Lagides pour les animaux exotiques nouvellement découverts et l'importation à Alexandrie de tels animaux. Ces animaux pouvaient être logés, stockés de façons très différentes. Ce qui importait, c'était moins l'unité de lieu que l'unité de temps d'une fête royale ou religieuse, qui fournissait l'occasion de montrer, au peuple et aux visiteurs venus du monde grec, les animaux capturés par les souverains. Cet aspect est plus radical encore dans le monde romain : si l'on excepte les singes et les oiseaux, utilisés comme animaux de compagnie, l'emploi principal des animaux exotiques était de participer à des spectacles violents qui se finissaient par leur mise à mort. Par ailleurs, on peut trouver toute une série de similitudes dans la fonction d'un côté du lieu zoo, de l'autre de ces manifestations, en particulier celle de l'extension d'un pouvoir de domination. Car les animaux les plus admirés dans l'Antiquité sont ceux réputés les plus sauvages, en particulier le tigre et l'éléphant. À cause de la conquête indienne d'Alexandre,

\footnotetext{
il y avait une association étroite entre la personne
}

du souverain, le charisme royal et le contrôle sur l'éléphant (la victoire sur l'Inde d'Alexandre est avant tout une victoire sur l'éléphant de guerre indien). Après la mort d'Alexandre, les Ptolémées n'ont pu se procurer des éléphants indiens parce que la route était coupée par l'empire séleucide. Ils ont donc exploré méthodiquement la mer Rouge, ont équipé ses côtes de stations de ravitaillement, ont créé beaucoup plus au Sud, des sortes de camps de base pour les chasseurs d'éléphants, conçu une logistique extrêmement impressionnante pour acheminer jusqu'à Memphis, jusqu'à Alexandrie, des éléphants africains dont l'existence était connue mais dont la domestication restait à faire! Cette infrastructure d'ampleur a donné l'impulsion au développement des relations commerciales en mer Rouge. Et donner lieu à, qui sait, un exemple de symétrie d'échange exotique ? On ne sait précisément, en effet, ce que donnaient les marchands méditerranéens en échange des biens exotiques qu'ils importaient de l'océan indien via la Mer Rouge. Du numéraire, du blé, du vin, de l'huile, des esclaves, cela est attesté. Mais l'archéologie a permis de rallonger la liste d'un produit courant et relativement peu apprécié côté méditerranéen: le corail rouge de Méditerranée et de mer Rouge. La valeur des perles des uns en échange de la valeur du corail des autres. 
Le désir détalonner le rapport à l'autre se manifesterait dans les combats organisés entre faune indigène et faune exotique; taureau contre rhinocéros, par exemple. Mais les animaux exotiques étant souvent plus puissants que les animaux indigènes, il semblerait que l'on n'ait pas accordé à ces derniers une dimension identitaire forte. Sinon leur déroute répétée serait fâcheuse et... de mauvaise augure !

Jean Trinquier 